\title{
Presentation, diagnosis, and treatment of a cerebellar tuberculoma: illustrative case
}

\author{
Stephen Capone, MD, ${ }^{1,2}$ Dokpe Emechebe, $M D,{ }^{3}$ Eric G. St. Clair, $M D,{ }^{2}$ Ali Sadr, $M D,{ }^{2}$ and Michelle Feinberg, $\mathrm{MD}^{2}$ \\ ${ }^{1}$ St. George's University School of Medicine, Grenada, West Indies; ${ }^{2}$ Department of Neurosurgery, Kings County Hospital Center, Brooklyn, New York; and ${ }^{3}$ Department of \\ Pathology, SUNY Downstate Medical Center, Brooklyn, New York
}

\begin{abstract}
BACKGROUND Central nervous system (CNS) tuberculomas are a feared complication of tuberculosis (TB) infection. These lesions can present in varying manners and are associated with significant morbidity and mortality. Prompt diagnosis and treatment of the lesion and the underlying infection are critical in the care of these patients. The authors presented a case of a 45-year-old Yemeni immigrant presenting with a 3-month history of severe right temporo-occipital headaches with photophobia and night sweats. Imaging showed a rim-enhancing lesion in the right cerebellar hemisphere.

OBSERVATIONS Laboratory tests were unremarkable and within normal limits. QuantiFERON testing was negative, ruling out latent TB infection. The patient received a suboccipital craniotomy, and resection of the cerebellar lesion showed caseating granuloma formation, which was positive for acidfast bacilli and Fite stain.
\end{abstract}

LESSONS CNS tuberculomas are an important differential to consider in patients with a history of primary TB, regardless of active disease or immunocompetent status. Resection of these lesions remains a viable treatment option that is safe and effective.

https://thejns.org/doi/abs/10.3171/CASE21170

KEYWORDS cerebellar; tuberculoma; infection; granuloma; tuberculosis

Although the incidence of tuberculosis (TB) remains low in developed countries, one of the feared complications is central nervous system (CNS) infection. CNS TB can present as tuberculous meningitis, osteomyelitis of the spine, or a distinct mass referred to as a tuberculoma, ${ }^{1}$ all of which contain active Mycobacterium tuberculosis and are highly contagious. These discrete tuberculoma lesions can present in unique manners, masquerading as hematomas, ${ }^{2}$ intracranial tumors, ${ }^{3,4}$ or multiple CNS lesions, ${ }^{5}$ and they are commonly found in children, immunosuppressed patients, or patients with active TB. ${ }^{6-8}$ On computed tomography (CT) and magnetic resonance imaging (MRI), tuberculomas typically appear as ring-enhancing or hyperdense lesions with associated edema, ${ }^{9,10}$ although distinctive imaging characteristics are not well established. Because of this, intracranial tuberculomas can be misdiagnosed as numerous other intracranial pathologies, particularly when there is a low suspicion of active TB or prior exposure.

The case rate of TB has decreased progressively over years, with fewer than 10,000 cases annually in the United States. ${ }^{11,12}$
Despite the declining rate in the United States, the disease burden of TB infection remains a concern across World Health Organization member states. ${ }^{12}$ Although rare, CNS TB accounts for approximately $1 \%-2 \%$ of all TB cases worldwide and can have significant morbidity and mortality. ${ }^{10}$ Here we present a rare case of a CNS tuberculoma, interestingly presenting in an immunocompetent patient without active pulmonary disease or latent infection.

\section{Illustrative Case \\ Presentation}

A 45-year-old male Yemeni immigrant presented to the Kings County Hospital Center emergency department in Brooklyn, New York, with a 3-month history of severe right temporo-occipital headaches with photophobia and night sweats. The patient denied fevers, cough, chest pain, shortness of breath, and weight loss. On presentation, the patient was neurologically intact and afebrile, and laboratory studies were unremarkable (Table 1). A head CT scan

ABBREVIATIONS AFB = acid-fast bacilli; CNS = central nervous system; CSF = cerebrospinal fluid; $C T$ = computed tomography; MRI = magnetic resonance imaging; NAAT = nucleic acid-based amplification technique; RIPE = isoniazid, rifampin, ethambutol, and pyrazinamide; TB = tuberculosis.

INCLUDE WHEN CITING Published November 1, 2021; DOI: 10.3171/CASE21170.

SUBMITTED June 28, 2021. ACCEPTED July 12, 2021.

(c) 2021 The authors, CC BY-NC-ND 4.0 (http://creativecommons.org/licenses/by-nc-nd/4.0/). 
TABLE 1. Laboratory results for blood tests on admission

\begin{tabular}{lc}
\hline \multicolumn{1}{c}{ Test } & Result \\
\hline Complete blood count & \\
\hline WBC & $7.49 \mathrm{k} / \mathrm{uL}$ \\
\hline HGB & $13.9 \mathrm{~g} / \mathrm{dL}$ \\
\hline HCT & $42.7 \%$ \\
\hline PLT & $177 \times 10^{9} / \mathrm{L}$ \\
\hline Neutrophil & $57.9 \%$ \\
\hline Lymphocyte & $24.2 \%$ \\
\hline Monocyte & $5.7 \%$ \\
\hline Eosinophil & $11.6 \%(\uparrow)$ \\
\hline Basophil & $0.5 \%$ \\
\hline Basic metabolic panel & \\
\hline Sodium & $140 \mathrm{mEq} / \mathrm{L}$ \\
\hline Potassium & $4.1 \mathrm{mEq} / \mathrm{L}$ \\
\hline Chloride & $104 \mathrm{mEq} / \mathrm{L}$ \\
\hline CO ${ }_{2}$ & $26 \mathrm{mEq} / \mathrm{L}$ \\
\hline Glucose & $102 \mathrm{mg} / \mathrm{dL}$ \\
\hline BUN & $20.0 \mathrm{mg} / \mathrm{dL}$ \\
\hline Creatinine & $0.92 \mathrm{mg} / \mathrm{dL}$ \\
\hline Calcium & $9.7 \mathrm{mg} / \mathrm{dL}$ \\
\hline Magnesium & $2.06 \mathrm{mEq} / \mathrm{L}$ \\
\hline Phosphorus & $3.5 \mathrm{mg} / \mathrm{dL}$ \\
\hline Hepatic function & \\
\hline Total bilirubin & $0.3 \mathrm{mg} / \mathrm{dL}$ \\
\hline AST & $94 \mathrm{units} / \mathrm{L}$ \\
\hline Albumin & $31 \mathrm{units} / \mathrm{L}$ \\
\hline Direct bilirubin & $5.0 \mathrm{~g} / \mathrm{dL}$ \\
\hline ALT & $7.4 \mathrm{~g} / \mathrm{dL}$ \\
\hline Phos & $<0.2 \mathrm{mg} / \mathrm{dL}$ \\
\hline units/L \\
\hline M
\end{tabular}

Alk Phos = alkaline phosphatase; $\mathrm{ALT}=$ alanine aminotransferase; $\mathrm{AST}=$ aspartate aminotransferase; $\mathrm{BUN}=$ bun urea nitrogen; $\mathrm{HGB}=$ hemoglobin; $\mathrm{HCT}=$ hematocrit; PLT = platelets; $\mathrm{WBC}=$ white blood cells.

showed a partially calcified $2.5 \times 2.0 \mathrm{~cm}$ extra-axial right cerebellar mass with adjacent vasogenic edema (Fig. 1). The differential included a calcified meningioma versus an atypical infectious process. MRI showed a heterogenous peripherally enhancing lesion in the posterior right cerebellar hemisphere with irregular margins and surrounding presumed vasogenic edema on both T1-weighted postcontrast and T2-weighted sequences (Fig. 2). There was no evidence of elevated cerebral blood volume on perfusion imaging but mild distortion of the fourth ventricle due to mass effect. Differential diagnosis included atypical infectious process, including parasitic or granulomatous infection, versus a brain neoplasm. At this stage, further examination of the mass was warranted. Because infection could not be ruled out, the patient received additional testing and tested negative for toxoplasma, cysticercosis, Aspergillus, and the presence of $(1,3)$-beta-D-glucans but he tested positive for schistosoma antibodies. QuantiFERON testing on hospital day 4 showed negative results, ruling out latent TB infection. ${ }^{13,14} \mathrm{CT}$ of the chest/ abdomen/pelvis showed no evidence of intrathoracic or abdominal
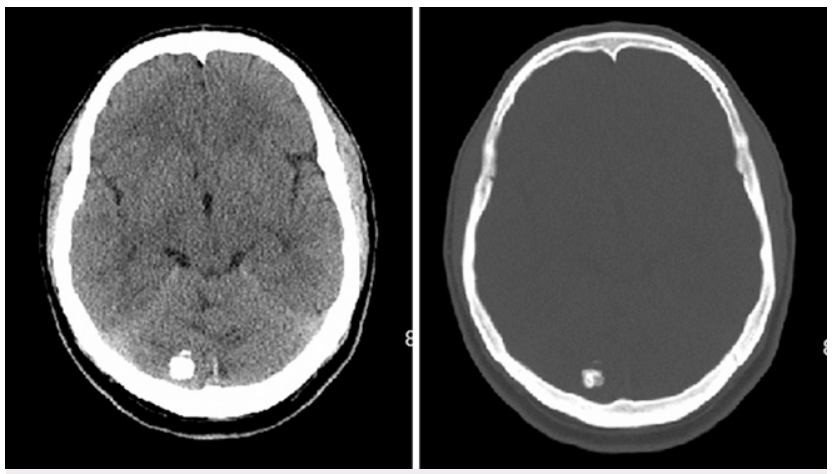

FIG. 1. Noncontrast head CT of the patient showing the brain (left) and bone (right) windows demonstrates a solitary calcified mass in the right cerebellar hemisphere with surrounding vasogenic edema.

metastasis but showed calcified mediastinal, left hilar, and mesenteric lymph nodes suggestive of prior granulomatous disease. Because of the risk associated with lumbar punctures with a posterior fossa mass, cerebrospinal fluid (CSF) analysis was not able to be performed. Treatment options were discussed with the patient, and he consented to resection of the mass.

\section{Treatment}

The patient received a right suboccipital craniotomy and resection of the mass. Intraoperatively, the mass was noted to have a soft tissue component with a large, calcified mass. Margins of the lesion were dissected, and circumferential exposure was completed. The mass was easily removed, and the resection cavity was copiously irrigated. Hemostasis was achieved, and dura was reapproximated before replacement of the bone flap and closure of the incision.

Postoperative imaging showed a gross total resection. Pathological staining of the specimen showed positive acid-fast bacilli (AFB) and Fite stains and necrotizing granulomatous inflammation, confirming the diagnosis of a tuberculoma (Fig. 3).
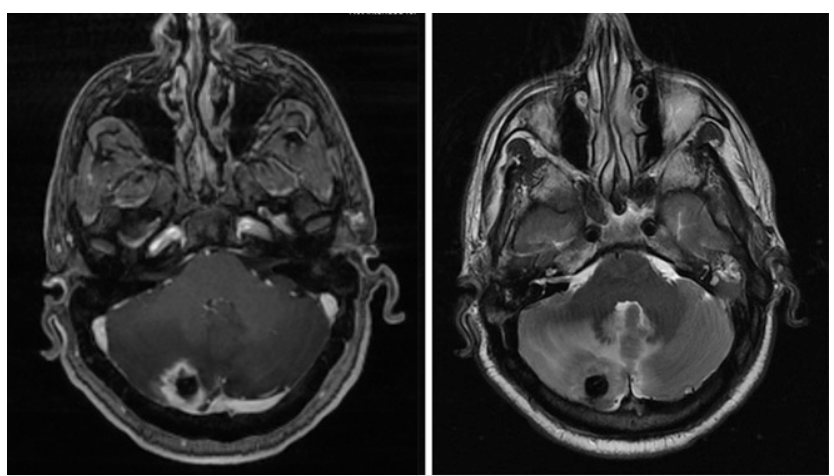

FIG. 2. Brain MRI performed preoperatively. T1 postcontrast imaging (left) demonstrates a heterogenous peripherally enhancing lesion in the posterior right cerebellar hemisphere with irregular margins and surrounding presumed vasogenic edema. T2 imaging (right) shows an isointense to mildly hyperintense signal with avid irregular enhancement. These findings are consistent with the posterior fossa calcified mass demonstrated on CT. 

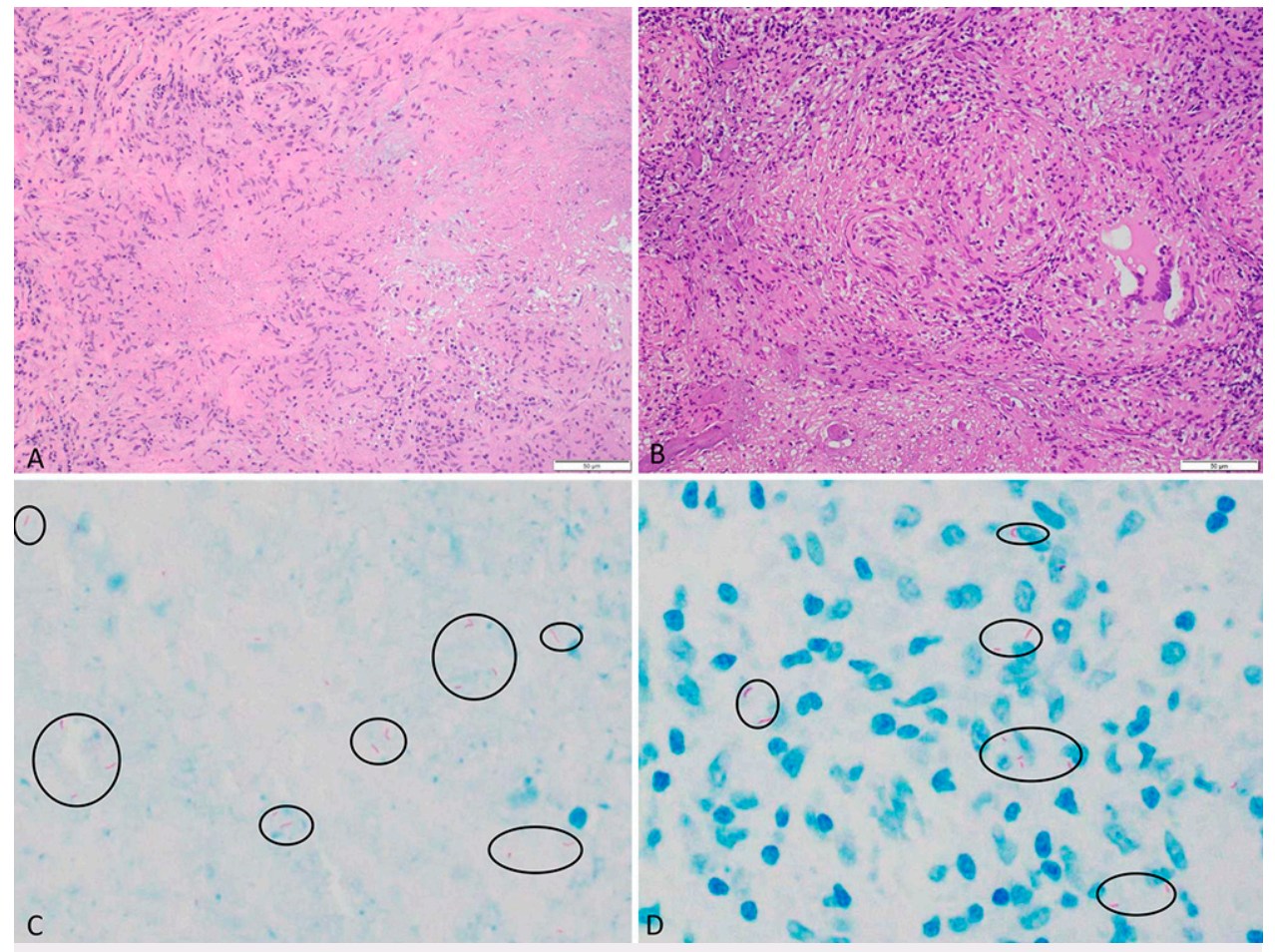

FIG. 3. A: Microscopically, hematoxylin and eosin stain of the lesion revealed areas of central necrosis surrounded by epithelioid cells and inflammatory cells (magnification $\times 100$ ). B: Other areas show multinucleated giant cells, epithelioid cells, and inflammatory cells consistent with necrotizing granuloma (magnification $\times 200)$. Staining for AFB $(\mathbf{C}$, magnification $\times 1,000)$ and FITE $(\mathbf{D}$, magnification $\times 1,000)$ are positive for acid-fast mycobacteria (black circles).

\section{Discharge}

The patient was discharged home on postoperative day 3 on multidrug treatment for TB, including rifampin, isoniazid, ethambutol, and pyrazinamide for 2 months followed by rifampin and isoniazid for an additional 10 months, all of which required direct observational therapy per state health department policy. At discharge, the patient was awake and alert and oriented to self, place, and time with a Glasgow Coma Scale score of 15 . His preoperative reports of headaches had completely resolved after surgery.

\section{Discussion}

\section{Observations}

CNS TB remains a concern for patients with prior exposure to Mycobacterium tuberculosis, both recent and remote. Additionally, patients on active TB treatment have presented with paradoxical CNS tuberculomas, despite decreasing disease burden in the lungs. ${ }^{10,15,16}$ Presenting symptoms for these caseating granulomas may vary but in general mimic those of other space-occupying intracranial lesions because they are generally secondary to the mass effect, edema, and subsequent increased intracranial pressure. These symptoms include papilledema, headache, nausea, ataxia, diplopia, and, if active TB is present, weight loss. ${ }^{1,4,6,17,18}$ When present in the cerebellum, these lesions carry a greater risk of obstructive hydrocephalus due to the decreased volume of the posterior fossa and proximity to the fourth ventricle and cerebral aqueduct.

Treatment options for intracranial tuberculomas remain limited and consist primarily of a four-medication anti-TB regimen of isoniazid, rifampin, ethambutol, and pyrazinamide (RIPE) with or without corticosteroids to treat the associated cerebral edema. ${ }^{9,10}$ Here we present a case showing efficacy of the adjunct use of resection of a solitary cerebellar tuberculoma without active or latent TB infection, leading to the cessation of symptoms and removal of the primary lesion. Resection of these lesions has been previously reported, ${ }^{2,8,15}$ particularly in lesions causing significant CNS symptoms and obstructive hydrocephalus or strongly mimicking other pathologies. Lesions present in the cerebellum may be more commonly resected because they are more likely to cause these severe symptoms. ${ }^{3,15,16}$ Care must be taken during surgery to minimize the spread of the infectious Mycobacteria because it may cause significant complications, in particular, TB meningitis. It is important to note that clinical decisions vary based on clinical picture, test results, and suspicion of CNS TB. The combination of negative serologies, no active disease, and a negative QuantiFERON test result led to the decision not to perform additional invasive testing, such as lumbar puncture and CSF analysis. Had the QuantiFERON or CT imaging showed positive results, the clinical decision to pursue further testing to confirm the diagnosis would have been favored. If this diagnosis had been confirmed by QuantiFERON or additional testing, we anticipate primary treatment would be the RIPE protocol. ${ }^{1,8,10}$ Had the patient then failed RIPE or had symptoms progressed, surgical treatment would have been considered. In cases of unconfirmed CNS TB, CSF analysis can yield valuable information, including Mycobacterium presence by AFB staining, a mononuclear pleocytosis, high protein, low glucose, and a "ground-glass" appearance. ${ }^{10}$ Given a questionable diagnosis and a 
CSF analysis meeting these criteria, CNS TB can be confirmed. ${ }^{10}$ CSF culture may also confirm the diagnosis but may delay treatment because growth requires 3 to 6 weeks. ${ }^{10}$ Additionally, the use of nucleic acid-based amplification technique (NAAT) is unclear, with the reliability of testing not established and a high rate of false-positive results. ${ }^{10}$ Given a high clinical suspicion of CNS TB in combination with negative AFB staining of the CSF, NAAT may be warranted, although a negative test result does not exclude the diagnosis. ${ }^{10}$

Of note, this patient was not immunosuppressed, had negative QuantiFERON test results, and did not have active pulmonary TB, all of which are commonly associated with the presentation of CNS tuberculomas. Presentation of a patient with a CNS TB infection prompts concurrent testing for immunosuppressive diseases because there have been reported cases of human immunodeficiency virus diagnosed secondary to CNS TB. ${ }^{19}$ Rarely, these cases can present in patients without immunosuppression. ${ }^{20}$ This case serves as a reminder that the differential diagnosis in patients with known prior TB exposure who present with an intracranial mass suspicious for infection should include CNS tuberculoma, regardless of time since exposure, presence or absence of active disease, results of QuantiFERON testing, and current immune state.

\section{Lessons}

We described a patient with a rare cerebellar tuberculoma without the presence of active disease. These CNS tuberculomas are an important differential to consider in patients with a history of primary TB, regardless of disease status. Resection of these lesions remains a viable treatment option that is safe and effective in conjunction with established anti-TB drug therapies.

\section{Acknowledgments}

We would like to thank St. George's University School of Medicine for their support of this research.

\section{References}

1. Chin JH. Neurotuberculosis: a clinical review. Semin Neurol. 2019; 39(4):456-461.

2. Ranjan M, Saritha A, Mahadevan A, Shankar SK, Sampath S. Cerebellar tuberculoma presenting as haematoma: a case report and pathophysiological consideration. Br J Neurosurg. 2009;23(2):203-205.

3. Binesh F, Taghipour Zahir S, Roshan Bovanlu T. Isolated cerebellar tuberculoma mimicking posterior cranial fossa tumour. Case Reports. 2013;2013:bcr2013009965.

4. Greschus S, Kuchelmeister K, Oeynhausen S, Fischer HP, Urbach $\mathrm{H}$. Cerebral tuberculoma mimicking brain tumor. Clin Neuroradiol. 2014;24(4):389-393.

5. Meegada S, Gyamfi R, Muppidi V, Dandu V, Challa T. Multiple intracranial tuberculomas with an intra-medullary spinal cord tuberculoma in a pediatric patient. Cureus. 2020;12(3):e7248.

6. Monteiro R, Carneiro JC, Costa C, Duarte R. Cerebral tuberculomas: a clinical challenge. Respir Med Case Rep. 2013;9(1):34-37.

7. Martínez-Vázquez C, Bordón J, Rodríguez-González A, et al. Cerebral tuberculoma: a comparative study in patients with and without HIV infection. Infection. 1995;23(3):149-153.
8. Zahrou F, Elallouchi Y, Ghannane H, Benali SA, Aniba K. Diagnosis and management of intracranial tuberculomas: about 2 cases and a review of the literature. Pan Afr Med J. 2019;34:23.

9. Moussa AA, Mahmoud ME, Yousef HA. Intracranial tuberculoma and recent advances in magnetic resonance imaging. Egypt $\mathrm{J}$ Neurosurg. 2018;33(1):13.

10. Leonard JM. Central nervous system tuberculosis. Microbiol Spectr. 2017;5(2):117278.

11. Centers for Disease Control and Prevention. Division of Tuberculosis Elimination. TB Incidence in the United States, 1953-2019. Accessed June 19, 2021. https://www.cdc.gov/tb/statistics/tbcases. $\mathrm{htm}$.

12. Langer AJ, Navin TR, Winston CA, LoBue P. Epidemiology of tuberculosis in the United States. Clin Chest Med. 2019;40(4):693-702.

13. Sester $M$, Sotgiu $G$, Lange $C$, et al. Interferon- $\gamma$ release assays for the diagnosis of active tuberculosis: a systematic review and metaanalysis. Eur Respir J. 2011;37(1):100-111.

14. Metcalfe JZ, Everett CK, Steingart KR, et al. Interferon- $\gamma$ release assays for active pulmonary tuberculosis diagnosis in adults in lowand middle-income countries: systematic review and meta-analysis. J Infect Dis. 2011;204(suppl 4):S1120-S1129.

15. Ninomiya A, Saito A, Ishida T, et al. A surgical case of cerebellar tuberculoma caused by a paradoxical reaction while on therapy for tuberculosis spondylitis. Surg Neurol Int. 2019;10(162):162.

16. Agrawal A, Kakani A, Mahajan SN, Vagha SJ, Acharya S. Paradoxical manifestation of cerebellar tuberculoma. Infect Dis Clin Pract. 2011;19(1):72-74.

17. Togha M, Sahraian MA, Hosseini SJ, Haddadi A. Brain tuberculoma: report of a case presented with prolonged nonspecific symptoms and multiple brain tuberculoma. Iran J Neurol. 2012;11(1): 34-35.

18. Kelly J, Kinirons M. Intracranial tuberculomas. Lancet. 2003; 362(9386):797.

19. Meltzer E, Moussawi K. Intracranial tuberculoma in a patient with untreated human immunodeficiency virus. JAMA Neurol. 2017; 74(3):356-357.

20. Matsumoto $\mathrm{Y}$, Aikawa $\mathrm{H}$, Narita $\mathrm{S}$, et al. Intracranial tuberculoma in non-immunosuppressive state. Neurol Med Chir (Tokyo). 2013;53(4):259-262.

\section{Disclosures}

The authors report no conflict of interest concerning the materials or methods used in this study or the findings specified in this paper.

\section{Author Contributions}

Conception and design: St. Clair, Feinberg Acquisition of data: Capone, St. Clair, Sadr, Feinberg Analysis and interpretation of data: Capone, Emechebe Drafting the article: Capone Critically revising the article: Capone, Emechebe, Feinberg Reviewed submitted version of manuscript: Capone, Feinberg Approved the final version of the manuscript on behalf of all authors: Capone Administrative/technical/ material support: St. Clair Study supervision: St. Clair, Sadr.

\section{Correspondence}

Stephen Capone: St. George's University School of Medicine, Grenada, West Indies. stephencaponemd@gmail.com. 